\title{
SOME RESULTS ON THE STABLE HOMOTOPY GROUPS OF SPHERES
}

\author{
BY JOEL M. COHEN ${ }^{1}$
}

\author{
Communicated by F. P. Peterson, March 22, 1966
}

In this paper, we examine a particular example of a long-known spectral sequence in order to answer the lowest dimensional unresolved question about the stable homotopy groups of spheres. In particular we completely determine the $p$-primary component of $\pi_{n}(S)$ for $n \leqq 2(p-1)\left(p^{2}+2 p\right)-6$, for odd primes $p$.

There is a spectral sequence [7] $\left\{E^{r}, d^{r}\right\}$ such that $E_{* *}^{2}$ $=H_{*}\left(X ; \pi_{*}(S)\right)$ and $E_{* *}^{\infty}$ is a bigraded group associated with the stable homotopy groups of $X$, any topological space. This spectral sequence may be generalized by replacing space $X$ by spectrum $\boldsymbol{A}: E_{* *}^{2}=H_{*}\left(\boldsymbol{A} ; \pi_{*}(\boldsymbol{S})\right)$ and $E_{* *}^{\infty}$ is a bigraded group associated with $\pi_{*}(A)$.

Consider this spectral sequence for $\boldsymbol{A}=\boldsymbol{K}(Z)$, the Eilenberg-MacLane spectrum $\left(A_{n}=K(Z, n)\right)$. Then $E_{0,0}^{\infty}=Z$ and $E_{s, t}^{\infty}=0$ for $(s, t)$ $\neq(0,0)$, because $\pi_{*}(K(Z))=\pi_{0}(K(Z))=Z$.

$E_{* *}^{2}=H_{*}\left(K(Z) ; \pi_{*}(S)\right)$ is a tensor and torsion product of a wellknown ring, $H_{*}(K(Z))[1]$, and a ring about which information is sought, $\pi_{*}(S)$. This relation gives us the information needed.

For the actual computations, we replace $S$ by the spectrum $L_{p}$ where $\left(L_{p}\right)_{n}=M\left(Z_{p}, n\right)$ is a Moore space of homology type $\left(Z_{p}, n\right)$. It is easily shown that $\pi_{n}\left(L_{p}\right) \cong \pi_{n}(S) \otimes Z_{p}+\operatorname{Tor}\left(\pi_{n-1}(S), Z_{p}\right)$, $p$ odd. Thus given any $\theta \epsilon \pi_{n}(S)$ not divisible by $p$ we have an element also called $\theta \epsilon \pi_{n}\left(L_{p}\right)$. Given any $\eta \epsilon \pi_{n}(S)$ of order $p$, we have an element $\eta^{\prime} \epsilon \pi_{n+1}\left(L_{p}\right)$. ( $\eta^{\prime}$ can be constructed using Toda's toral construction [6] and is defined up to indeterminacy $\pi_{n+1}(S) \otimes Z_{p} \subset \pi_{n+1}\left(L_{p}\right)$.)

Multiplication can be defined making $\pi_{*}\left(L_{p}\right)$ an algebra over $Z_{p}$. Then $E_{* *}^{\infty}=E_{0,0}^{\infty}=Z_{p}$ and

$$
E_{* *}^{2}=H_{*}\left(K(Z) ; \pi_{*}\left(L_{p}\right)\right)=H_{*}\left(K(Z) ; Z_{p}\right) \otimes \pi_{*}\left(L_{p}\right)=A_{*} \otimes \pi_{*}\left(L_{p}\right)
$$

where $A_{*}$ is isomorphic to $E\left(\tau_{1}, \tau_{2}, \cdots\right) \otimes P\left(\xi_{1}, \xi_{2}, \cdots\right)$, the exterior algebra on generators $\tau_{i}$ tensored with the polynomial algebra on generators $\xi_{i}$, where $\operatorname{dim} \tau_{i}-1=\operatorname{dim} \xi_{i}=2\left(p^{i}-1\right) . A_{*}$ is the dual algebra to the quotient coalgebra of the Steenrod Algebra, by the left ideal generalized by the Bockstein.

1 These results are part of the author's doctoral dissertation submitted to the Massachusetts Institute of Technology. The author wishes to thank Professors D. W. Anderson and G. W. Whitehead for their advice and guidance. 
Toda [5] had found elements $\alpha_{n} \epsilon_{p} \pi_{2 n(p-1)-1}(S)$ where $\alpha_{1}$ is in the image of the $J$-homomorphism and $\left\langle\alpha_{r}, p_{i}, \alpha_{s}\right\rangle=\alpha_{r+s}$. He also found the elements $\beta_{s} \epsilon_{p} \pi_{2(s p+s-1)(p-1)-2}(S)$ with $\beta_{1}=\left\langle\alpha_{1}, \alpha_{1}, \cdots(p) \cdots, \alpha_{1}\right\rangle$. He proved that $\beta_{1}^{p} \alpha_{1} \neq 0$ if and only if there is some nonzero element $\gamma \epsilon_{p} \pi_{2 p^{2}(p-1)-2}(S)$. We prove that $\beta_{1}^{p} \alpha_{1} \neq 0$, and combining this with some further advances of May [3] we have the following partial description of ${ }_{p} \pi_{*}(S)$ :

THEOREM. The $p$-primary component of $\pi_{n}(S)$ for $0<n \leqq\left(p^{2}+2 p\right) q-6$ (where $q=2(p-1)$ ) is described completely by the following table listing each element, its order, and its dimension: (In this table $j \geqq 1$.)

Element Order Dimension

$\alpha_{j} \quad p \quad j q-1, j \neq \equiv 0(\bmod p)$

$\begin{array}{lll}\alpha_{j p}^{(2)} & p^{2} & j p q-1, j \neq \equiv 0(\bmod p) \\ \alpha_{j p^{2}}^{(3)} & p^{3} & j p^{2} q-1, j \neq \equiv 0(\bmod p)\end{array}$

$\beta_{m+1} \beta_{1}^{j-1} \quad p \quad((j+m) p+m) q-2 j, 0 \leqq m \leqq p-2$

$\alpha_{1} \beta_{m+1} \beta_{1}^{j-1} \quad p \quad((j+m) p+m+1) q-2 j-1,0 \leqq m \leqq p-2$

$\gamma \quad p \quad p^{2} q-2$

$\beta_{m+1} \beta_{1}^{j-1} \gamma \quad p \quad\left(p^{2}+j p+m p+m\right) q-2 j-2,0 \leqq m \leqq p-2$, $j=1$, if $m=1$ and $p=3$

$\alpha_{1} \beta_{m+1} \beta_{1}^{j-1} \gamma \quad p \quad\left(p^{2}+j p+m p+m+1\right) q-2 j-3,0 \leqq m \leqq p-2$, $j=1$, if $m=1$ and $p=3$

$\begin{array}{lll}\alpha_{m} \gamma & p & \left(p^{2}+m\right) q-3,1 \leqq m \leqq p-2 \\ \epsilon_{m} & p & \left(p^{2}+m\right) q-2,1 \leqq m \leqq p-1 \\ \phi & p^{2} & \left(p^{2}+p\right) q-3\end{array}$

$\beta_{2} \beta_{p-1} \beta_{1}^{j-1} \quad p \quad\left(p^{2}+j p+p-1\right) q-2 j-2 ; j=1$ if $p=3$.

If we consider the above terms as being in $\pi_{*}(S) \otimes Z_{p} \subset \pi_{*}\left(L_{p}\right)$, then we have the following relations: (Let $\alpha=\alpha_{1}$.)

$$
\begin{aligned}
\alpha_{n} & =\left(\alpha^{\prime}\right)^{n-1} \alpha, \quad \alpha_{n p}=\left(\alpha^{\prime}\right)^{n p-1} \alpha, \alpha_{n p^{2}}=\left(\alpha^{\prime}\right)^{n p^{2}-1} \alpha, n \neq 0(\bmod p) \\
\epsilon_{m} & =\left(\alpha^{\prime}\right)^{m} \gamma+m\left(\alpha^{\prime}\right)^{m-1} \alpha \gamma^{\prime}, \quad 1 \leqq m \leqq p-1, \\
\beta_{1}^{\prime} \beta_{p-1}^{\prime} & =\epsilon_{p-2} .
\end{aligned}
$$


Furthermore, there are homotopy operations $T$ and $R$ such that $T\left(\beta_{i}\right)$ $=\beta_{i+1}, 1 \leqq i \leqq p-2$ and $R\left(\beta_{1}\right)=\gamma$. (Equalities hold for a particular choice of basis elements.)

REMARKs. By Toda [5], our result is equivalent to the following: Given $N$, consider the sequence of topological spaces $K_{1} \subset K_{2} \subset \ldots$ $\subset S^{N}$ where $\pi_{j}\left(K_{k}\right)=0$ for $j \geqq N+k$ and the inclusion $i: S^{N} \rightarrow K_{k}$ induces $i_{*}: \pi_{j}\left(S^{N}\right) \approx \pi_{j}\left(K_{k}\right)$ for $j<N+k$. Let $A^{i}\left(K_{k} ; Z_{p}\right)=H^{N+i}\left(K_{k} ; Z_{p}\right)$ for $0 \leqq i<N+k$. (This does not depend on $N$.) There is a generator $b_{p} \in A^{k+1}\left(K_{k} ; Z_{p}\right)$ for $k=\left(p^{2}-1\right) q-2$. Then $\mathcal{P}^{1} b_{p} \in A^{k+2 p-1}\left(K_{k} ; Z_{p}\right)$. Toda proves [5] that $\rho^{1} b_{p}=0$ if and only if $\beta^{p} \alpha_{1} \neq 0$. Thus it follows from our result that $P^{1} b_{p}=0$.

BRIEF OUTLINE OF PROOF. We use the fact that $d^{r}$ is known to be a derivation. The first nonzero element in positive dimension in the base is $\xi_{1}$ in $E_{q, 0}^{2}$. Since it cannot be a boundary, it must be a noncycle. Hence there must be some nonzero element in $E_{0, q-1}^{2} \cong \pi_{q-1}\left(L_{p}\right)$. This element must be the image of $\alpha_{1}$. Thus $d^{q}\left(\xi_{1}\right)=\alpha_{1}$. Then $d^{q}\left(\xi_{1}^{2}\right)$ $=2 \xi_{1} \alpha_{1}, \cdots, d^{q}\left(\xi_{1}^{p-1}\right)=-\xi_{1}^{p-2} \alpha_{1}$, so that all these elements are cancelled (i.e. are boundaries or noncycles. But then $d^{q}\left(\xi_{1}^{p}\right)=p \xi_{1}^{p-1} \alpha_{1}=0$. Thus $\xi_{1}^{p-1} \alpha_{1}$ is not a boundary hence it must be a noncycle. Thus there must be a nonzero element in $E_{0, p q-2}^{2} \cong \pi_{p q-2}\left(L_{p}\right)$. (This element is $\beta_{1}$.) In exactly the same way, $\xi_{1}^{p-1} \beta_{1}^{i} \alpha_{1}$ is a nonboundary hence $\beta_{1}^{i+1} \neq 0$, $i \leqq p-1$. Then we assume that $\beta_{1}^{p} \alpha_{1}=0$. There is nothing natural to cancel $\beta_{1}^{p+1}$ (which May [3] proves is nonzero). Thus it becomes increasingly difficult to assure that each noncycle is a boundary, and finally we see that there is an infinite cycle which is not a boundary. This contradicts the fact that $E_{s, t}^{\infty}=0$ if $(s, t) \neq(0,0)$, so the assumption is incorrect and $\beta_{1}^{p} \alpha_{1} \neq 0$.

Detailed proofs and a more complete statement of our results will appear elsewhere.

\section{REFERENCES}

1. H. Cartan et al., Algèbres d'Eilenberg-MacLane et homotopie, Séminaire Henri Cartan de l'École Normale Supérieure, 17 année, (1954/55), 2eme ed., Secrétariat mathématique, 11 rue Pierre Curie, 1956.

2. S. Gitler and J. D. Stasheff, The first exotic class of BF, Topology 4 (1965), 257-266.

3. J. P. May, The cohomology of the Steenrod algebra, stable homotopy groups of spheres, Bull. Amer. Math. Soc. 71 (1965), 377-380.

4. N. E. Steenrod and D. B. A. Epstein, Cohomology operations, Princeton Univ. Press, Princeton, N. J., 1962.

5. H. Toda, p-primary components of homotopy groups: III, Stable groups of the sphere, Mem. Coll. Sci., Univ. Kyoto 31 (1958), 191-210. 
6. - Composition methods in homotopy groups of spheres, Princeton Univ. Press, Princeton, N. J., 1962.

7. G. W. Whitehead, Generalized homology theories, Trans. Amer. Math. Soc. 102 (1962), 227-283.

Massachusetts Institute of Technology 